\title{
Outcomes of percutaneous cholecystostomy in elderly patients: a systematic review and meta-analysis
}

\author{
George Markopoulos, Francesk Mulita, Dimitris Kehagias, Stylianos Tsochatzis, Charalampos Lampropoulos, \\ loannis Kehagias \\ Department of Surgery, General University Hospital of Patras, Patras, Greece
}

Gastroenterology Rev 2021; 16 (3): 188-195

DOI: https://doi.org/10.5114/pg.2020.100658

Key words: percutaneous cholecystostomy, cholecystitis, elderly.

Address for correspondence: Dr. Francesk Mulita, Department of Surgery, General University Hospital of Patras, Greece,

e-mail: oknarfmulita@hotmail.com

\begin{abstract}
Introduction: Percutaneous cholecystostomy (PC) represents a management option to control sepsis in patients with acute cholecystitis, who are unable to tolerate surgery.

Aim: This review aimed to evaluate the outcomes of elderly patients treated with PC and compare it with emergent cholecystectomy.

Material and methods: An electronic search of the Embase, Medline Web of Science, and Cochrane databases was performed. Percutaneous cholecystostomy was used as the reference group, and weighted mean differences (WMD) were calculated for the effect of PC on continuous variables, and pooled odds ratios (POR) were calculated for discrete variables.

Results: There were 20 trials included in this review. Utilisation of PC was associated with significantly increased mortality $(\mathrm{POR}=4.85 ; 95 \% \mathrm{Cl}: 1.02-7.30 ; p=0.0001)$ and increased re-admission rates (POR $=2.95 ; 95 \% \mathrm{Cl}: 2.21-3.87 ; p<0.0001)$.

Conclusions: This pooled analysis established that patients treated with PC appear to have increased mortality and readmission rates relative to those managed with cholecystectomy.
\end{abstract}

\section{Introduction}

Acute cholecystitis represents a common general surgical emergency and is one of the most frequent complications of gallstones requiring inpatient hospital admission. Definitive treatment requires cholecystectomy to prevent further gallstone-related complications. Operative intervention during the initial hospital admission has been associated with improved patient outcomes with decreased long-term complications, shorter overall length of stay, and reduced overall healthcare costs [1-4]. However, in critically unwell patients who may not be able to tolerate surgery, percutaneous cholecystostomy represents a management option to control sepsis. This procedure involves a drainage tube being inserted through the skin into the gallbladder, usually under image guidance. This procedure aims to drain the gallbladder and relieve sepsis in those patients who are not considered suitable for emergency cholecystectomy, and it provides a clinical improvement in over $85 \%$ of patients within $48-72 \mathrm{~h}$ of the intervention [5]. Percu- taneous cholecystostomy is particularly utilised in highrisk patients such as those being treated in intensive care or the elderly. Increasing age significantly reduces the likelihood of emergency cholecystectomy being undertaken [6, 7], in part due to the reduced physiological reserve of these individuals and the associated increase in peri-operative risks. This can lead to increased utilisation of percutaneous cholecystostomy in elderly patients who are deemed unfit for cholecystectomy.

The use of percutaneous cholecystostomy in critically unwell patients is reflected in the Tokyo Guidelines for management of acute cholecystitis [8, 9], which recommend that in patients with evidence of organ dysfunction (grade III cholecystitis) percutaneous cholecystostomy can be utilised to drain the gallbladder and control sepsis prior to interval cholecystectomy. However, only around $40 \%$ of patients receiving percutaneous cholecystostomy will go on to receive definitive cholecystectomy within 1 year [10]. Of those patients treated with percutaneous cholecystostomy alone, up to $46 \%$ develop further episodes of cholecystitis within 3 years [11]. 


\section{Aim}

This systematic review and meta-analysis aimed to evaluate the outcomes of elderly patients treated with percutaneous cholecystostomy versus treatment with emergent cholecystectomy.

\section{Material and methods}

An electronic literature search was undertaken using the Embase, Medline, and Web of Science databases up to May 2020. The search terms 'cholecystostomy' and 'percutaneous cholecystostomy' and the Medical Subject Heading (MeSH) 'cholecystostomy' (MeSH) were used in combination with the Boolean operators AND or OR. Three authors (ST, CL, DK) performed the electronic search independently in May 2020. The electronic search was supplemented by a hand-search of published abstracts from relevant meetings. The reference lists of articles obtained were also searched to identify further relevant citations. Abstracts of the articles identified by the electronic search were scrutinised to determine their suitability for inclusion in the pooled analysis.

Publications were included if they were case-matched controlled or comparative studies in which patients underwent percutaneous cholecystostomy or emergency cholecystectomy for acute cholecystitis. Studies were excluded if they were non-comparative or investigated alternative management strategies such as delayed cholecystectomy. Because this review aimed to specifically investigate the utilisation of percutaneous cholecystostomy in elderly patients, studies were also excluded if the mean age in the percutaneous cholecystostomy group was below 65 years.

\section{Outcome measures}

Primary outcome measures were mortality (in-hospital and 30-day) and overall morbidity (defined as a complication developing within 30 days of the procedure - either percutaneous cholecystostomy or cholecystectomy). Secondary outcome measures were length of hospital stay and readmission rate.

\section{Statistical analysis}

Data from eligible studies was entered into a computerized spreadsheet for analysis. Statistical analysis was performed using StatsDirect 3.2.9 (StatsDirect, Altrincham, UK). Percutaneous cholecystostomy was utilised as the reference group. Weighted mean difference (WMD) was calculated for the effect of emergency cholecystectomy on continuous variables. Pooled odds ratios (POR) were calculated for the effect of emergen- cy cholecystectomy on discrete variables. All pooled outcome measures were determined using random-effects models as described by DerSimonian Laird [12]. Heterogeneity among trials was assessed by means of the Cochran's Q statistic, a null hypothesis in which $p<0.05$ is taken to indicate the presence of significant heterogeneity [13]. The Egger test was used to assess the funnel plot for significant asymmetry as an indication of possible publication or other biases.

\section{Results}

The literature search identified 19 case-matched control or comparative studies and one randomised control trial, which were included for analysis [14-33]. Figure 1 shows the PRISMA flowchart for the literature search. In total there were 689,874 patients included, with 28,241 patients receiving percutaneous cholecystostomy. There were 662,327 patients treated by other means in the comparison group, with 661,633 undergoing cholecystectomy and 694 having been treated conservatively in one study, which also included patients managed with emergency cholecystectomy [17].

Table I describes patient demographics in each included study. Table II shows the results from the individual studies for the clinical outcome measures evaluated in this pooled analysis.

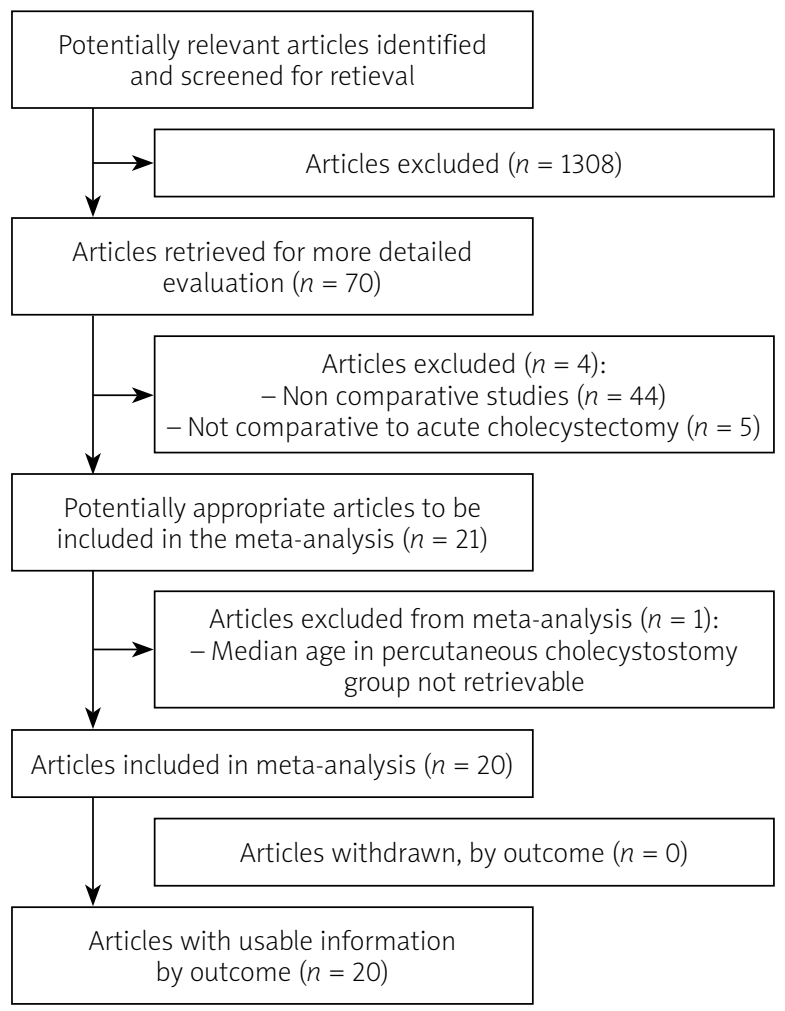

Figure 1. PRISMA flow chart of literature search 


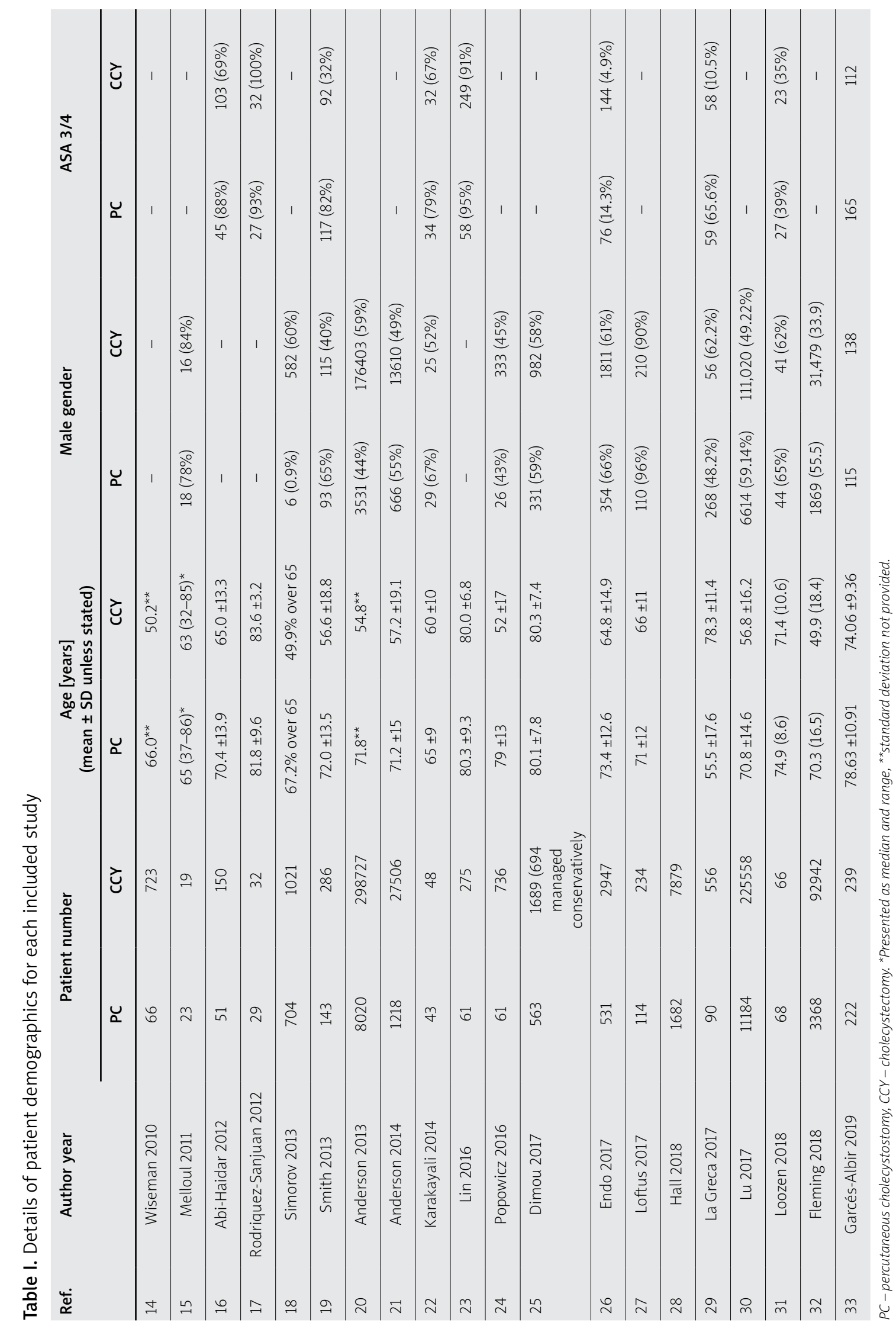




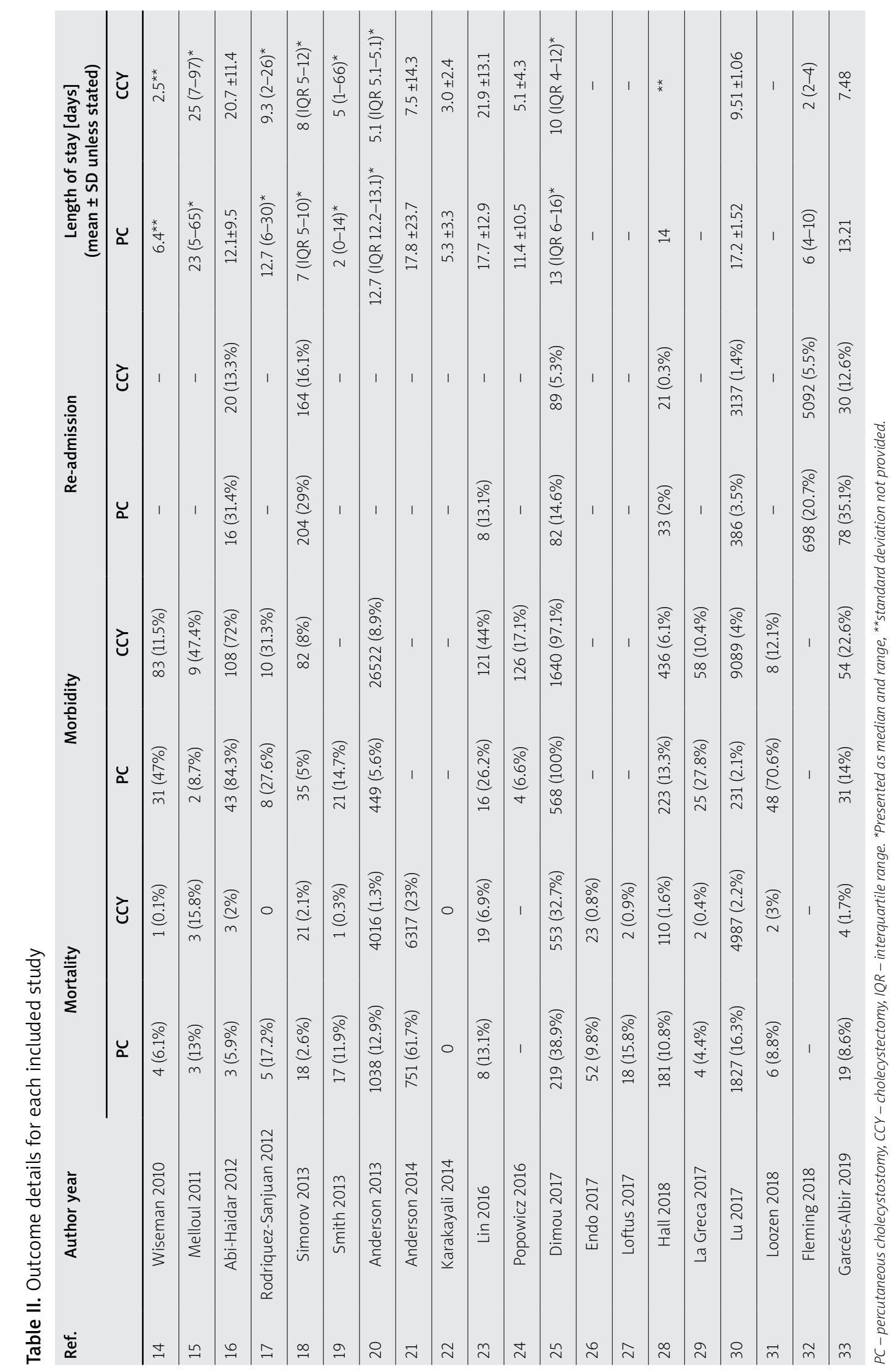




\section{Mortality}

The incidence of mortality was demonstrated by 17 studies [14-23, 25-27, 29-31, 33]. The utilisation of percutaneous cholecystostomy was associated with a significant increase in mortality $(\mathrm{POR}=4.85 ; 95 \% \mathrm{Cl}$ : $1.02-7.30 ; p=0.0001$ ) (Figure 2). There was proof of statistical heterogeneity (Cochran $\mathrm{Q}=698.55 ; p<0.0001$; $P^{2}=97.3 \%$ ), but no evidence of bias (Egger $=-2.67$; $p=0.41)$.

\section{Overall morbidity}

Overall morbidity was studied in 13 studies [14-18, $20,23-25,29-31,33]$. There was no significant difference in overall morbidity between percutaneous cholecystostomy and emergency cholecystectomy $(\mathrm{POR}=0.92 ; 95 \% \mathrm{Cl}: 0.45-1.98 ; p=0.83)$. There was proof of statistical heterogeneity (Cochran $Q=237.02$; $p<0.0001 ; I^{2}=93,7 \%$ ), but no evidence of bias (Egger $=-2.06 ; p=0.33$ ).

\section{Secondary outcome measures}

Three studies stated rate of readmission [14, 17, 23]. Percutaneous cholecystostomy was associated with a significantly increased rate of readmission (POR $=2.95 ; 95 \% \mathrm{Cl}: 2.21-3.87 ; p<0.0001)$ (Figure 3). There was no proof of significant heterogeneity (Cochran Q 10.44; $p=0.21 ; l^{2}=33.2 \%$ ).

Eleven studies reported length of stay [14-24]. There was no significant difference in length of stay between the two groups (pooled weighted mean difference: 0.47 ;
95\% Cl: -1.74 to $2.92 ; p=0.64)$. There was evidence of heterogeneity (Cochran $\mathrm{Q}=18654.23 ; p<0.0001$; $\left.p^{2}=100 \%\right)$, but no proof of bias (Egger $=-18.97 ; p=0.21$ ).

\section{Discussion}

This systematic review and meta-analysis highlights that patients treated with percutaneous cholecystostomy appear to have increased mortality and readmission rates relative to those treated with cholecystectomy on index admission with acute cholecystitis.

Previous studies identified that undertaking cholecystectomy for elderly patients during initial hospital admission prevents further episodes of gallstone-related disease, reduces readmission rates, and is associated with lower overall healthcare costs [34]. The study by Riall et al. identified that $38 \%$ of elderly patients who did not receive cholecystectomy on primary hospital admission would be re-admitted with gallstone-related readmission within 2 years, compared to a $4 \%$ readmission rate for those undergoing cholecystectomy [34].

In order to extend the benefits of acute cholecystectomy to critically unwell patients who are deemed unfit for surgery, previous studies have suggested utilising percutaneous cholecystostomy as a 'bridging-procedure' prior to early cholecystectomy within a few days $[35,36]$. This management strategy allows sepsis to be resolved by decompression of the gallbladder using percutaneous cholecystostomy, and one study reported clinical resolution of toxaemia within $24 \mathrm{~h}$ in $85 \%$ of patients following percutaneous cholecystostomy placement [35]. Following resolution of sepsis, patients

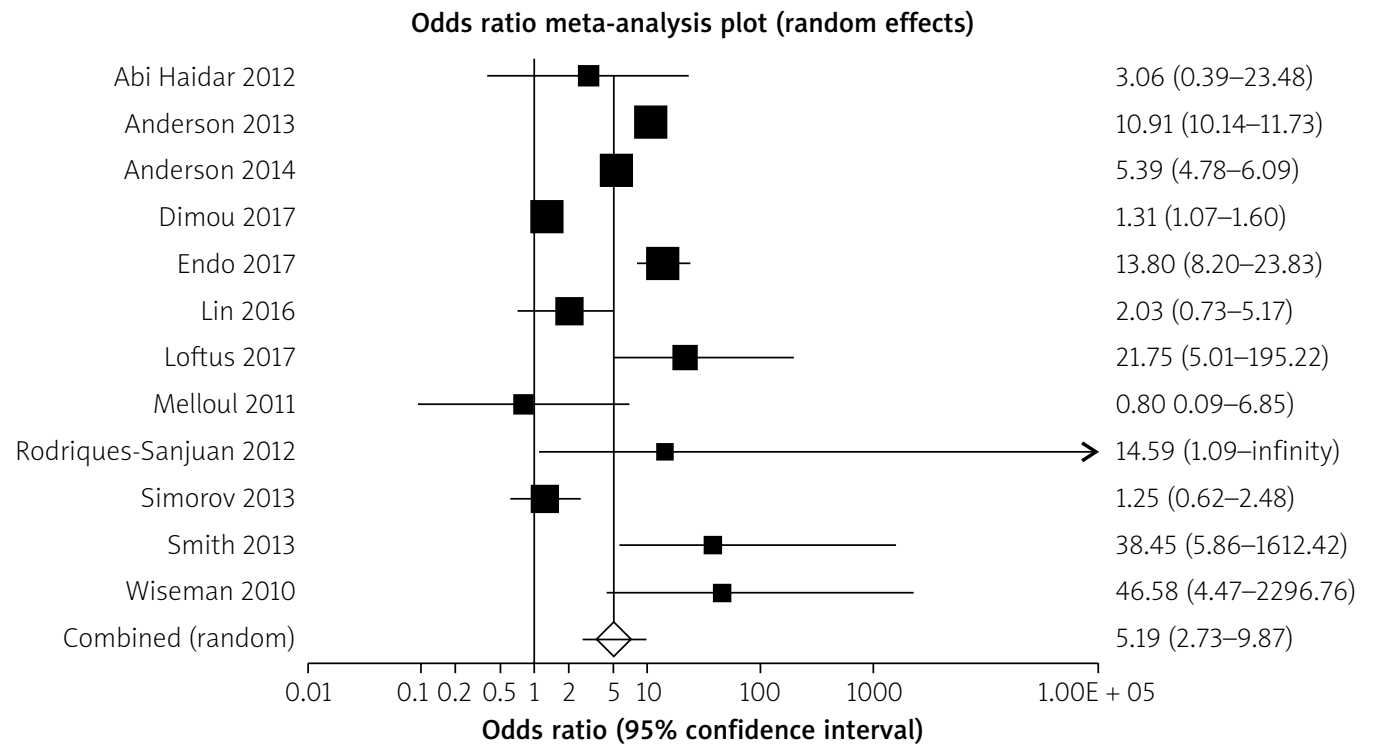

Figure 2. Percutaneous cholecystostomy associated with significantly increased mortality $(P O R=4.85$, 95\% Cl: $1.96-8.30)$ 


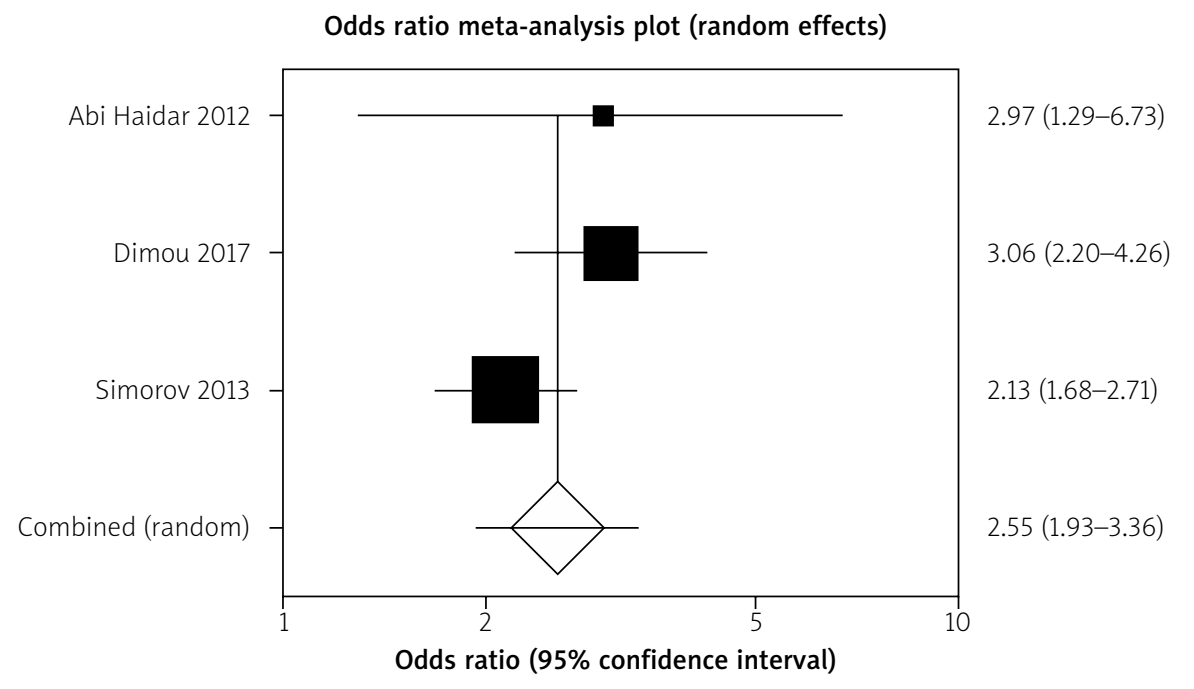

Figure 3. Percutaneous cholecystostomy associated with significantly increased rate of readmission $(\mathrm{POR}=2.95,95 \% \mathrm{Cl}: 2.21-3.87)$

may be more likely to be considered as candidates for surgery.

The laparoscopic approach for cholecystectomy is known to provide significant advantages to patients in terms post-operative recovery [37]. There have been concerns that undertaking delayed cholecystectomy following the prior placement of a percutaneous cholecystostomy can be associated with increased conversion from laparoscopic to open surgery, with some previous studies reporting conversion rates as high as $32-43 \%$ [38-40]. However, when utilising percutaneous cholecystostomy as a bridge to surgery, the aforementioned studies reported laparoscopic completion rates of $87.7 \%$ and $93.5 \%$ when cholecystectomy was completed within 5 days of $P C$ placement $[35,36]$.

To date, there has been no published randomised controlled trials (RCT) that have compared the utilisation of percutaneous cholecystostomy with conservative management in acute cholecystitis. There is only one, recently published, RCT that compared percutaneous cholecystostomy with laparoscopic cholecystectomy in the emergency setting [31]. The present pooled analysis is based mainly upon non-randomised data, and therefore there are important limitations that must be considered in the interpretation of the results gained. The Tokyo guidelines recommend the utilisation of percutaneous cholecystostomy only in patients who have severe grade III cholecystitis with evidence of organ dysfunction [8, 9]. Those patients receiving percutaneous cholecystostomy may have more been more significantly unwell during the acute episode than those deemed suitable for surgical treatment with cholecystectomy.
This factor may have been partially responsible for the increased rates of mortality with percutaneous cholecystostomy identified in the current pooled analysis.

The multicentre RCT was concluded early after a planned interim analysis, because laparoscopic cholecystectomy compared with percutaneous catheter drainage was proven to reduce the rate of major complications. The rate of death did not differ between the laparoscopic cholecystectomy and percutaneous catheter drainage group [31]. This general outcome is the exact opposite of the pooled analysis findings of the included studies in this review. The early termination of the RCT and the subsequent small number of patients in comparison with the other observational studies may be responsible for this discrepancy in the results. One other limitation in comparing the results of this RCT with the current meta-analysis is the different definition of mortality (1 year versus 30 days). However, this study provides a more robust evaluation of the potential benefits of laparoscopic cholecystectomy in high-risk patients with acute cholecystitis.

Only one study in the present pooled analysis included patients who had been managed conservatively $(n=694)$ as well as those managed with cholecystectomy $(n=995)$ in the reference group [25]. This formed part of a propensity-matched analysis with and without percutaneous cholecystostomy tube placement in grade III cholecystitis. It was not possible to separate the outcome data for patients who underwent cholecystectomy and those managed by conservative means for this study. This study provided important information regarding readmission rates following percutaneous 
cholecystostomy or alternative management strategies. Therefore, it was decided to include this study in the pooled analysis despite the fact that not all patients in the control group had received cholecystectomy.

It is difficult to determine the relative effectiveness of PC with or without delayed cholecystectomy compared to early cholecystectomy due to selection bias in favour of patients treated with PC. Other limitations of the current analysis include the fact that there was a significant amount of statistical heterogeneity in the analysis for mortality, morbidity, and hospital stay. This is likely to have been secondary to the variations in study populations for articles included in this pooled analysis.

\section{Conclusions}

This pooled analysis has established that patients treated with percutaneous cholecystostomy appear to have increased mortality and readmission rates compared to those managed with cholecystectomy. However, the utilisation of percutaneous cholecystostomy remains a valuable clinical tool in patients who are unfit for surgical intervention. Where possible, percutaneous cholecystostomy should be considered as a 'bridge to surgery', with cholecystectomy being undertaken following resolution of sepsis and patient stabilisation.

\section{Conflict of interest}

The authors declare no conflict of interest.

\section{References}

1. Sutton AJ, Vohra RS, Hollyman M, et al. Cost-effectiveness of emergency versus delayed laparoscopic cholecystectomy for acute gallbladder pathology. Br J Surg 2017; 104: 98-107.

2. Gurusamy K, Samraj K, Gluud C, et al. Meta-analysis of randomized controlled trials on the safety and effectiveness of early versus delayed laparoscopic cholecystectomy for acute cholecystitis. Br J Surg 2010; 97: 141-50.

3. Kolla SB, Aggarwal S, Kumar A, et al. Early versus delayed laparoscopic cholecystectomy for acute cholecystitis: a prospective randomized trial. Surg Endosc 2004; 18: 1323-7.

4. Wu XD, Tian X, Liu MM, et al. Meta-analysis comparing early versus delayed laparoscopic cholecystectomy for acute cholecystitis. Br J Surg 2015; 102: 1302-13.

5. Winbladh A, Gullstrand P, Svanvik J, et al. Systematic review of cholecystostomy as a treatment option in acute cholecystitis. HPB 2009; 11: 183-93.

6. CholeS Study Group, West Midlands Research Collaborative. Population-based cohort study of variation in the use of emergency cholecystectomy for benign gallbladder diseases. Br J Surg 2016; 103: 1716-26.

7. Bergman S, Sourial N, Vedel I, et al. Gallstone disease in the elderly: are older patients managed differently? Surg Endosc 2011; 25: 55-61.
8. Hirota M, Takada T, Kawarada Y, et al. Diagnostic criteria and severity assessment of acute cholecystitis: Tokyo Guidelines. J Hepatobiliary Pancreat Surg 2007; 14: 78-82.

9. Yokoe M, Takada T, Strasberg SM, et al. TG13 diagnostic criteria and severity grading of acute cholecystitis (with videos). J Hepatobiliary Pancreat Sci 2013; 20: 35-46.

10. de Mestral C, Gomez D, Haas B, et al. Cholecystostomy: a bridge to hospital discharge but not delayed cholecystectomy. J Trauma Acute Care Surg 2013; 74: 175-80.

11. Ha JPY, Tsui KK, Tang CN, et al. Cholecystectomy or not after percutaneous cholecystostomy for acute calculous cholecystitis in high-risk patients. Hepatogastroenterology 2008; 55 : 1497-502.

12. DerSimonian R, Laird N. Meta-analysis in clinical trials. Control Clin Trials 1986; 7: 177-88.

13. Higgins JPT, Thompson SG. Quantifying heterogeneity in a meta-analysis. Stat Med 2002; 21: 1539-58.

14. Wiseman JT, Sharuk MN, Singla A, et al. Surgical management of acute cholecystitis at a tertiary care center in the modern era. Arch Surg 2010; 145: 439-44.

15. Melloul E, Denys A, Demartines N, et al. Percutaneous drainage versus emergency cholecystectomy for the treatment of acute cholecystitis in critically ill patients: does it matter? World J Surg 2011; 35: 826-33.

16. Abi-Haidar Y, Sanchez V, Williams SA, et al. Revisiting percutaneous cholecystostomy for acute cholecystitis based on a 10-year experience. Arch Surg 2012; 147: 416-22.

17. Rodríguez-Sanjuán JC, Arruabarrena A, Sánchez-Moreno L, et al. Acute cholecystitis in high surgical risk patients: percutaneous cholecystostomy or emergency cholecystectomy? Am J Surg 2012; 204: 54-9.

18. Simorov A, Ranade A, Parcells J, et al. Emergent cholecystostomy is superior to open cholecystectomy in extremely ill patients with acalculous cholecystitis: a large multicenter outcome study. Am J Surg 2013; 206: 935-41.

19. Smith TJ, Manske JG, Mathiason MA, et al. Changing trends and outcomes in the use of percutaneous cholecystostomy tubes for acute cholecystitis. Ann Surg 2013; 257: 1112-5.

20. Anderson JE, Chang DC, Talamini MA. A nationwide examination of outcomes of percutaneous cholecystostomy compared with cholecystectomy for acute cholecystitis, 1998-2010. Surg Endosc 2013; 27: 3406-11.

21. Anderson JE, Inui T, Talamini MA, et al. Cholecystostomy offers no survival benefit in patients with acute acalculous cholecystitis and severe sepsis and shock. J Surg Res 2014; 190: 517-21.

22. Karakayali FY, Akdur A, Kirnap M, et al. Emergency cholecystectomy vs percutaneous cholecystostomy plus delayed cholecystectomy for patients with acute cholecystitis. Hepatobiliary Pancreat Dis Int 2014; 13: 316-22.

23. Lin WC, Chang CW, Chu CH. Percutaneous cholecystostomy for acute cholecystitis in high-risk elderly patients. Kaohsiung J Med Sci 2016; 32: 518-25.

24. Popowicz A, Lundell L, Gerber P, et al. Cholecystostomy as bridge to surgery and as definitive treatment or acute cholecystectomy in patients with acute cholecystitis. Gastroenterol Res Pract 2016; 2016: 3672416.

25. Dimou FM, Adhikari D, Mehta HB, et al. Outcomes in older patients with grade iii cholecystitis and cholecystostomy tube 
placement: a propensity score analysis. J Am Coll Surg 2017; 224: 502-511.e1.

26. Endo I, Takada T, Hwang TL, et. al. Optimal treatment strategy for acute cholecystitis based on predictive factors: JapanTaiwan multicenter cohort study. J Hepatobiliary Pancreat Sci 2017; 24: 346-61.

27. Loftus TJ, Collins EM, Dessaigne CG, et al. Percutaneous cholecystostomy: prognostic factors and comparison to cholecys tectomy. Surg Endosc 2017; 31: 4568-75.

28. Hall BR, Armijo PR, Krause C, et al. Emergent cholecystectomy is superior to percutaneous cholecystostomy tube placement in critically ill patients with emergent calculous cholecystitis. Am J Surg 2018; 216: 116-9.

29. La Greca A, Di Grezia M, Magalini S, et. al. Comparison of cholecystectomy and percutaneous cholecystostomy in acute cholecystitis: results of a retrospective study. Eur Rev Med Pharmacol Sci 2017; 21: 4668-74.

30. Lu P, Chan CL, Yang NP, et al. Outcome comparison between percutaneous cholecystostomy and cholecystectomy: a 10-year population-based analysis. BMC Surg 2017, 17: 130.

31. Loozen CS, van Santvoort HC, van Duijvendijk P, et al. Laparoscopic cholecystectomy versus percutaneous catheter drainage for acute cholecystitis in high risk patients (CHOCOLATE) multicentre randomised clinical trial. BMJ 2018; 363: k3965.

32. Fleming MM, DeWane MP, Luo J, et al. A propensity score matched comparison of readmissions and cost of laparoscopic cholecystectomy vs percutaneous cholecystostomy for acute cholecystitis. Am J Surg 2019; 217: 83-9.

33. Garcés-Albir M, Martín-Gorgojo V, Perdomo R, et al. Acute cholecystitis in elderly and high-risk surgical patients: is percutaneous cholecystostomy preferable to emergency cholecystectomy? J Gastrointest Surg 2019; DOI: 10.1007/s11605-01904424-5.

34. Riall TS, Zhang D, Townsend CM, et al. Failure to perform cholecystectomy for acute cholecystitis in elderly patients is associated with increased morbidity, mortality, and cost. J Am Col Surg 2010; 210: 668-77, 677-9.

35. Akyürek N, Salman B, Yüksel O, et al. Management of acute calculous cholecystitis in high-risk patients. Management 2005; 15: 315-20.

36. Macrì A, Scuderi G, Saladino E, et al. Acute gallstone cholecystitis in the elderly. Surg Endosc 2006; 20: 88-91.

37. Coccolini F, Catena F, Pisano M, et al. Open versus laparoscopic cholecystectomy in acute cholecystitis. Systematic review and meta-analysis. Int J Surg 2015; 18: 196-204.

38. Suzuki K, Bower M, Cassaro S, et al. Tube cholecystostomy before cholecystectomy for the treatment of acute cholecystitis. JSLS 2015; 19: e2014.00200.

39. McKay A, Abulfaraj M, Lipschitz J. Short- and long-term outcomes following percutaneous cholecystostomy for acute cholecystitis in high-risk patients. Surg Endosc 2012; 26: 1343-51.

40. Morse BC, Smith JB, Lawdahl RB, et al. Management of acute cholecystitis in critically ill patients: contemporary role for cholecystostomy and subsequent cholecystectomy. Am Surg 2010; 76: 708-12.

Received: 13.09 .2020

Accepted: 8.10.2020 